\title{
Study on the Parameter Optimization of Soft-switching DC/DC Converters with the Response Surface Methodology, a SPICE Model, and a Genetic Algorithm
}

\author{
Shuai Liu ${ }^{*}$, Li Wei ${ }^{\dagger}$, Yicheng Zhang ${ }^{*}$, and Yongtao Yao* \\ ${ }^{*}$ College of Electronics and Information Engineering, Tongji University, Shanghai, China
}

\begin{abstract}
The application of soft-switching techniques is increasing in the DC/DC converter area. It is important to design soft-switching parameters to ensure the converter operates properly and efficiently. An optimized design method is presented in this paper. The objective function is the total power loss of a converter, while the variables are soft-switching parameters and the constraints are the electrical requirements for soft-switching. Firstly, a response surface methodology (RSM) model with a high precision is built, and the rough optimized parameters can be obtained with the help of a genetic algorithm (GA) in the solution space determined by the constraints. Secondly, a re-optimization is conducted with a SPICE model and a GA, and accurate optimized parameters can be obtained. Simulation and experiment results show that the proposed method performs well in terms of a wide adaptability, efficiency, and global optimization.
\end{abstract}

Key words: GA, Parameters design optimization, RSM, Soft-switching converter, SPICE model

\section{INTRODUCTION}

Soft-switching techniques, with the characteristics of reducing switching loss, stress, and electro-magnetic interference (EMI), have been widely used in DC/DC applications [1]-[13]. The basic method for soft-switching is reshaping the voltage and current waveforms of the switches during switching intervals, by means of the resonance between the auxiliary inductor and the capacitor. This is done in order to reduce or even eliminate the overlapping area in the hard-switching mode. The evaluations of soft-switching converter performance in previous studies are generally based on soft-switching effects, i.e. as long as the overlapping area in soft-switching mode is smaller than that in the hard-switching mode, the soft-switching converter is believed to be effective. In addition, efficiency curves of the converter in different modes are provided, which can also demonstrate its

Manuscript received Oct. 10, 2014; accepted Jan. 12, 2015

Recommended for publication by Associate Editor Joung-Hu Park.

${ }^{\dagger}$ Corresponding Author: weili@tongji.edu.cn

Tel: +86-69585452, Tongji University

${ }^{*}$ College of Electronics and Information Eng., Tongji University, China effectiveness.

It is well known that auxiliary inductors and capacitors play an important role in soft-switching, and the selection of their values influences the final effects. Actually, there exists a concept of a soft-switching operation area. In other words, the determined range (i.e. the range of electrical parameters such as the input line, output load, etc.) where soft-switching can be achieved under the conditions of certain soft-switching parameters (i.e. the values of the auxiliary inductors and capacitors). In addition, there exists a concept of a soft-switching parameter area (as shown in Fig. 1, where $L_{r}$ is the value of the auxiliary inductor and $C_{r}$ is that of the auxiliary capacitor), which means that each group of parameters in this area can guarantee that the converter achieves soft-switching (at each soft-switching operation point).

Conventionally, the parameter area can be obtained according to the electrical characteristics of the converter (such as the rise rate of the auxiliary inductor current when turning on, the energy transfer relationship, etc.). However, there is no unified standard to screen out an accurate value (or point). Most researchers make this decision on the basis of engineering experience [1]-[10], while some (e.g. K. M. Smith, 


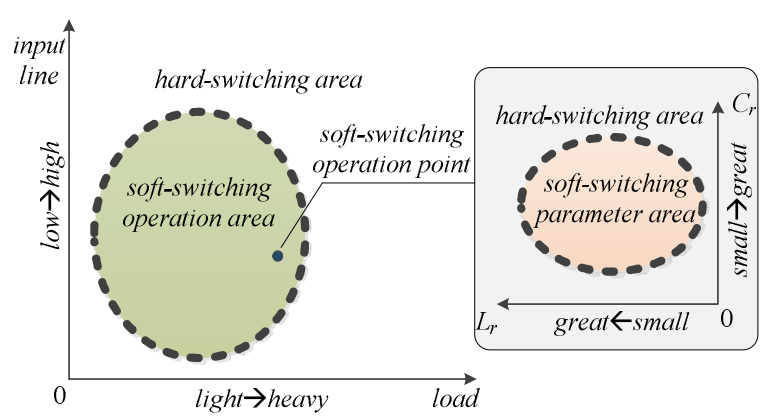

Fig. 1. Soft-switching operation and parameter area.

Jr.) takes advantage of the quantified switching loss [11]-[13].

K. M. Smith, Jr.'s method is as follows: firstly, obtain a large area of soft-switching parameters based on the electrical characteristics, whose members can guarantee the soft-switching effects; secondly, narrow this large area into a medium sized one based on the switching losses calculated with mathematical models, whose members can lower the switching losses; then, narrow the medium sized area to a small one based on the switching losses in the experiments, whose members are more reliable; finally, a group of accurate values can be selected from this small area. As described above, K. M. Smith, Jr. uses a step approximation method to approach the optimum values of parameters.

In previous studies, the method of parameter design according to engineering experience can be classified as heuristic search [14]-[16], which is effective for some specific topologies. However, it possesses bad portability and is not fit for other topologies. K. M. Smith, Jr.'s method is more systematic, while the disadvantages are: firstly, mathematical models of switching loss are ideal ones, which cannot describe the non-linear details of actual converters that well; secondly, K. M. Smith, Jr. uses the exhaustion method to obtain the small area, however, limited samples may result in an insufficient search of the solution space, as well as trapping in a local optimum; thirdly, only the losses from a switching operation not including the passive devices, line impedance, etc. is considered.

In this paper, an optimized design method of a RSM-SPICEGA is proposed. By using the quantified total power loss as a design criterion, the theory drawback of the engineering experience method is overcome. By using the global searching capability of a GA, the insufficient searching drawback of K. M. Smith, Jr.'s method is surmounted. By using a SPICE model, the nonlinear details of the switching devices can be shown to be as sufficient as possible. By using an RSM model, the calculation efficiency can be improved.

The rest of this paper is organized as follows. Section II discusses a mathematical description of the soft-switching parameter optimization. Section III describes the design solution of the RSM-SPICE-GA method, including the theory and flowchart, the selection of the RSM model, and the design of the optimization algorithm. Section IV presents simulation and experiment results of an example. Section V concludes the whole paper.

\section{MATHEMATICAL DESCRIPTION OF THE SOFT-SWITCHING PARAMETER OPTIMIZATION}

A mathematical model of the soft-switching parameter optimization can be described as follows:

\section{A. Objective Function and Optimization Variables}

The objective function is:

$$
\min P_{\text {loss }}\left(L_{r}, C_{r}\right), \quad m, n \in N
$$

where, $P_{\text {loss }}$ denotes the total power loss of the converter; $L_{r}=$ $\left\{L_{r l}, L_{r 2}, \ldots, L_{r m}\right\}$ denotes a m-dimensional optimization variable which represents a value set of the auxiliary inductors; and $C_{r}=\left\{C_{r l}, C_{r 2}, \ldots, C_{r n}\right\}$ denotes a n-dimensional optimization variable which represents a value set of the auxiliary capacitors. In this paper, only the relationship between the total power loss and the values of the auxiliary inductor and capacitor is concerned. Therefore, $P_{\text {loss }}$ is described as a function related to $L_{r}$ and $C_{r}$. Actually, $P_{\text {loss }}$ is also related to the other circuit parameters such as the input line, the output load, the value of the main inductor, etc.

\section{B. Constraints}

The constraint only related to the value of the auxiliary inductor is:

$$
h\left(L_{r i}\right) \geq 0, \quad i=1,2, \cdots, m
$$

The constraint only related to the value of the auxiliary capacitor is:

$$
g\left(C_{r j}\right) \geq 0, \quad j=1,2, \cdots, n
$$

The constraint related to the value of both the auxiliary inductor and the capacitor is:

$$
\begin{aligned}
& k\left(L_{r i}\right)+l\left(C_{r j}\right) \geq 0, \\
& i=1,2, \ldots, m, j=1,2, \ldots, n
\end{aligned}
$$

where, $h\left(L_{r i}\right)$ and $k\left(L_{r i}\right)$ represent functions that are only related to the value of the auxiliary inductor, while $g\left(C_{r j}\right)$ and $l\left(C_{r j}\right)$ represent functions only related to the value of the auxiliary capacitor. These constraints can be designed in accordance with the electrical requirements of the auxiliary inductor and capacitor during soft-switching operation (e.g. the resonant period, the turn-on and turn-off time, etc.).

The analysis above shows that the optimization of the soft-switching parameters can be converted into a nonlinear programming problem, whose objective function is the total power loss of the converter, the optimization variables are the soft-switching parameters, and the constraints are the electrical requirements for soft-switching.

\section{DESIGN OF THE RSM-SPICE-GA SOLUTION}

\section{A. Description of the Solution}




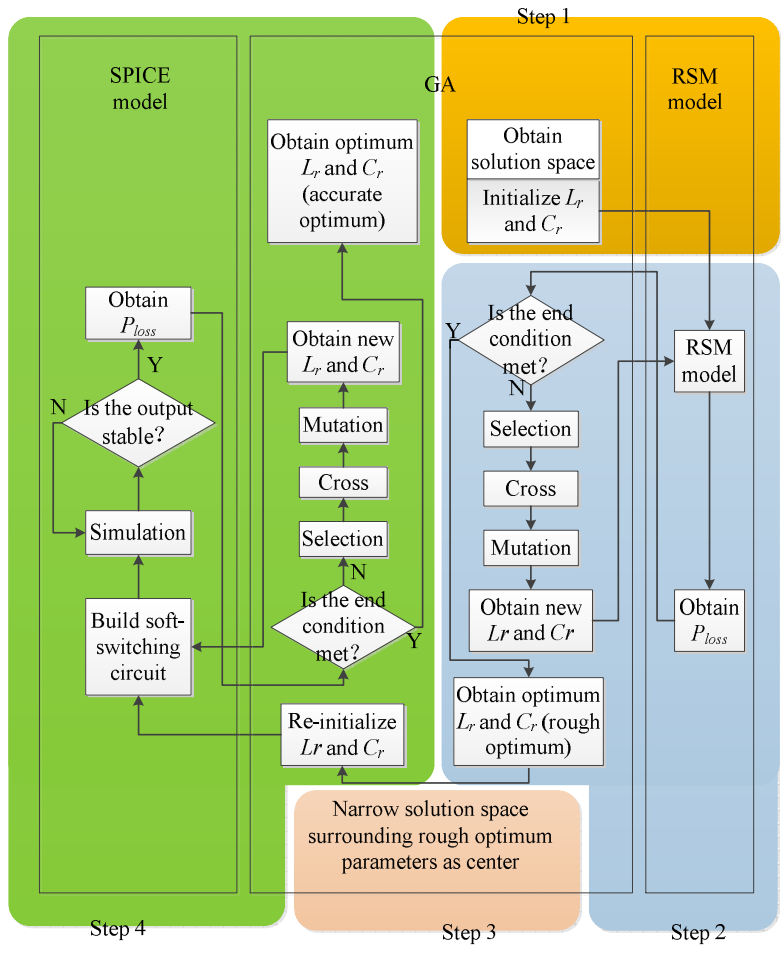

Fig. 2. Flowchart of RSM-SPICE-GA method.

A flowchart of the proposed method is shown in Fig. 2. Firstly, a large solution space is obtained by means of the constraints; secondly, a RSM model with Latin hypercube sampling (LHS) is built in this space, from which the power loss of different conditions can be calculated, then rough optimum soft-switching parameters can be obtained (i.e. rough location) with a GA; thirdly, surrounding the rough location, the solution space is narrowed to be a smaller one; finally, accurate optimum soft-switching parameters can be obtained with the help of a SPICE model and a GA (i.e. accurate location).

As the solution space determined by the constraints generally is a large one, searching in it is likely to result in staying around a locally optimal solution for long time, which will result in a waste of resources and even a reduction in obtaining the globally optimal solution. The method for narrowing the solution space is able to ensure that a globally optimal solution is obtained with a high probability, on the premise of unchanged algorithm accuracy and iterations. An important prerequisite for this method is accuracy of the RSM model, which is used in rough location. If the model cannot embody the characteristics of the soft-switching circuit well, the reliability and dependability of the rough location will decline drastically.

\section{B. Selection and Design of the Power Loss Model}

Mostly, the power loss model can be divided into an analytical model based on mathematical calculations [11]-[13] and a simulation model based on the physical structure

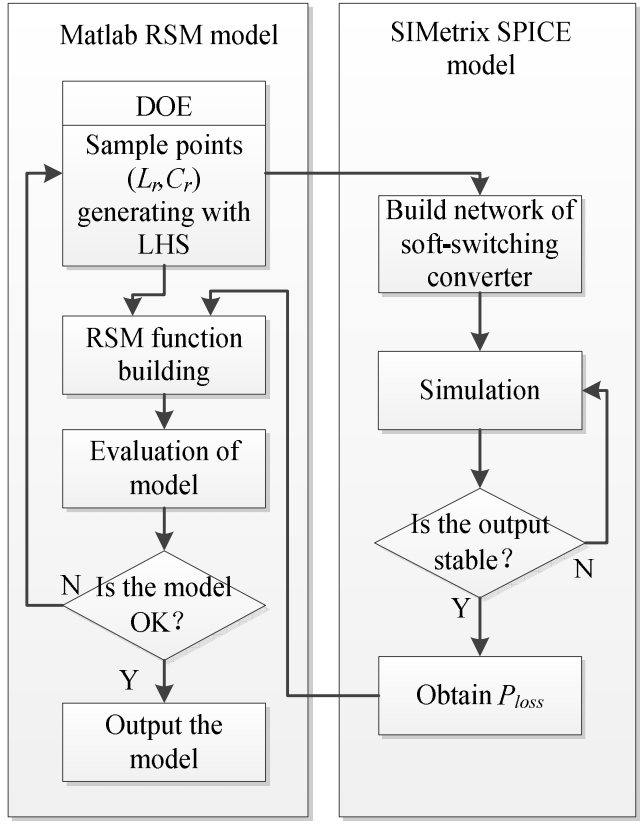

Fig. 3. Flowchart of RSM modeling.

[17][18]. The analytical model has rigorous reasoning, a distinct relationship and wide versatility, while it inevitably suffers from linearization errors due to the linear approximation of nonlinear problems, as well as errors due to circuit stray parameters. The simulation model (e.g. SPICE model) has a good mechanism and flexibility, embodies the characteristics of the devices totally and meticulously, but suffers from increased calculation time.

Considering the advantages and disadvantages of the two models, this paper introduces the concept of a RSM on the basis of a simulation model. The RSM was first proposed by Box and Wilson in 1951. It is an optimization method that combines experiment design and mathematical statistics. It can simplify complicated models through quantitative designing of the relationship between the variables and objective functions, and has good robustness [19]. In this paper, a RSM model is established, on the basis of experimental data from a simulation model. The modeling process, as shown in Fig. 3, is divided into three parts: the design of experiments (DOE), function building, and model evaluation.

1) Design of Experiments (DOE): Using the Model-Based Calibration toolbox of MATLAB, the Latin hypercube sampling (LHS) method is adopted in the DOE. With the principle of equal probability random orthogonal distribution, this method possesses the advantage of space filling to provide enough useful information [20].

2) Function Building: The conventional RSM function is a first-order or second-order polynomial, which does not fit in this case since a soft-switching converter is a strongly nonlinear system. An Augmented Radial Basis Function (ARBF) is adopted, consisting of Radial Basis Function (RBF) and a B-spline function, as follows [19]: 


$$
F(\boldsymbol{X})=\sum_{i=1}^{n} \lambda_{i} \varphi\left(\left\|\boldsymbol{X}-\boldsymbol{X}_{i}\right\|\right)+\sum_{j=1}^{m} p_{j}(\boldsymbol{X}) b_{j}
$$

where, $F(\boldsymbol{X})$ is estimated value of the ARBF, $\boldsymbol{X}$ is an unknown point, $\quad \boldsymbol{X}_{i}=\left(x_{1}, x_{2}, \ldots, x_{d}\right), i=1,2, \ldots, n \quad$ are d-dimensional interpolation sample points, $\lambda_{i}$ and $b_{j}$ are interpolation coefficients, $\left\|\boldsymbol{X}-\boldsymbol{X}_{i}\right\|$ is the Euclidean distance between two points, $\varphi$ is the basis function (a Multi-Quadric function is adopted here), and $\sum_{j=1}^{m} p_{j}(\boldsymbol{X}) b_{j}$ is the m-terms polynomial. Since it is hard to fit a strongly nonlinear problem in wide space, this paper adopts B-spline interpolation, by dividing the solution space into several small areas and fitting in the distribution, in order to improve the approximation quality of the model.

3) Model Evaluation: $\mathrm{R}^{2}$, adjusted $\mathrm{R}^{2}$ and the RMSE (Root Mean Square Error) are selected as criteria when evaluating the fitting accuracy.

\section{Design of the Optimization Algorithm}

This paper designs two optimization processes: one is a rough optimization with a RSM model (i.e. RSM-GA), and the other is an accurate optimization with a SPICE model (i.e. SPICE-GA). Both of them adopt a GA as the calculation tool. 1) Flowchart of the GA: The flowchart of the GA is shown in Fig. 2. Groups of variables are regarded as individuals, then go through selection, cross and mutate. The optimum one will be obtained in the end.

2) Realization of the Algorithm: The structure of the GA is shown as follows:

a) Population Initialization: Instead of simple random generation, this paper generates both random and space filling initial values with the LHS method. This ensures normal soft-switching operation and avoids calculations on unreasonable operating conditions. This increases the algorithm efficiency and covers all of the normal operating conditions as much as possible.

b) Coding Method: The real-number coding method is selected, because the variables are values of the auxiliary inductor and capacitor with physical meanings. Real-number coding is able to ensure a one-to-one correspondence between the genes in individuals and variables, increasing the convenience of the GA. Furthermore, some of the problems in the binary coding method (e.g. Hamming Cliff) can also be avoided. Nevertheless, given the physical meanings owned by the variables, they are not continuous but discrete after coding. This meets standards of the real inductor and capacitor, and simplifies the calculation.

c) Constraints and Fitness Function: When a soft-switching converter is operating, there exist some electrical requirements for the rate of $d v / d t$ and $\mathrm{di} / \mathrm{dt}$, the soft-switching intervals, the energy transfer relationship, etc., which can be used as

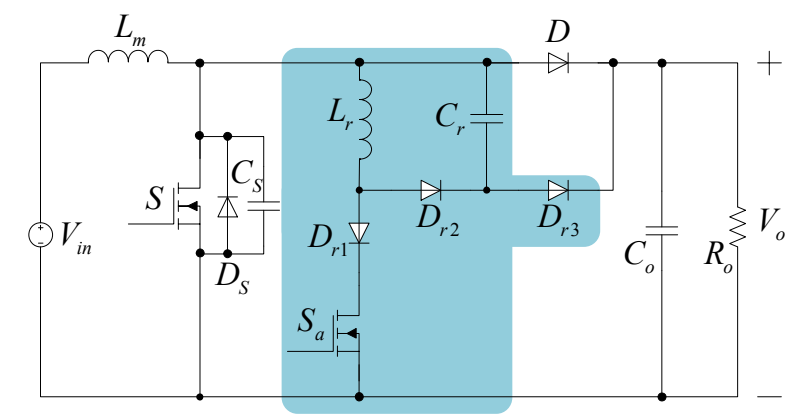

Fig. 4. Topology used in example.

constraints of optimization. The general forms are shown as Eqs. (2)-(4).

The fitness function can be expressed in the form of an augmented objective function, shown as Eq. (6), where $P_{\text {loss }}\left(L_{r}, C_{r}\right)$ is the objective function, $M$ is the penalty coefficient, $p\left(L_{r}, C_{r}\right)$ is the penalty term, and the latter two form a penalty function. From the penalty term, it is easy to know that the objective function (i.e. $P_{\text {loss }}$ ) will suffer an additional penalty when the variables (i.e. $L_{r}, C_{r}$ ) go out of the constraints scope. The individual selection probability is determined by the individual fitness value, where the smaller the value is, the higher the probability will be.

$$
\begin{aligned}
F( & \left.L_{r}, C_{r}, M\right) \\
\quad & P_{\text {loss }}\left(L_{r}, C_{r}\right)+M p\left(L_{r}, C_{r}\right) \\
& =P_{\text {loss }}\left(L_{r}, C_{r}\right)+ \\
& M\left\{\sum_{i=1}^{m} \min ^{2}\left[0, h\left(L_{r i}\right)\right]+\sum_{j=1}^{n} \min ^{2}\left[0, g\left(C_{r j}\right)\right]+\right. \\
& \left.\sum_{i=1, j=1}^{m, n} \min ^{2}\left[0, k\left(L_{r i}\right)+l\left(L_{r j}\right)\right]\right\}
\end{aligned}
$$

d) Selection Operator: The proportional selection and optimal individual reservation strategies are used in this paper, in order to avoid losing optimal individuals in the evolutionary process and lowering the global searching ability. This also improves the algorithm convergence.

e) Cross and Mutation Operator: The arithmetic cross method is adopted because of real-number coding, while a uniform mutation operator is used during mutation.

f) Improvement of the Convergence Criterion: If only the maximum number of generations (MAXGEN) is selected as criterion for the evolution end, the evolution will continue even if optimal solution appears long before MAXGEN. This would result in a waste of resources and a lowering of the algorithm efficiency. Therefore, both minimum reservation generations of the optimal individual and MAXGEN are adopted as criterion for the evolution end in this paper, which avoids the defect of single factor control criterion [21].

\section{ANALYSIS OF THE EXAMPLE}

A PWM Boost ZVT soft-switching converter [22] is selected 


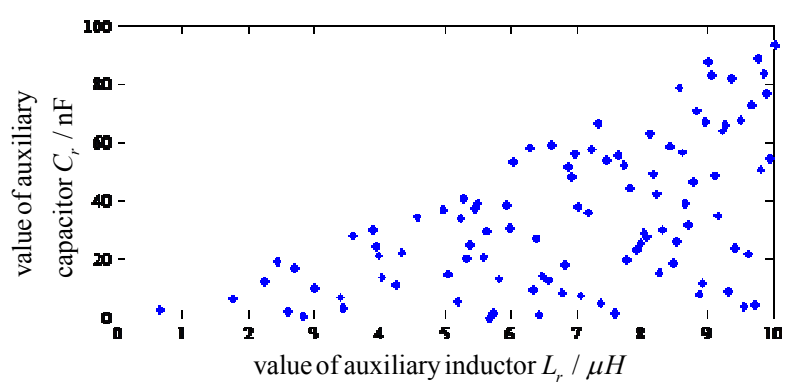

Fig. 5. Distribution of experimental sample points.

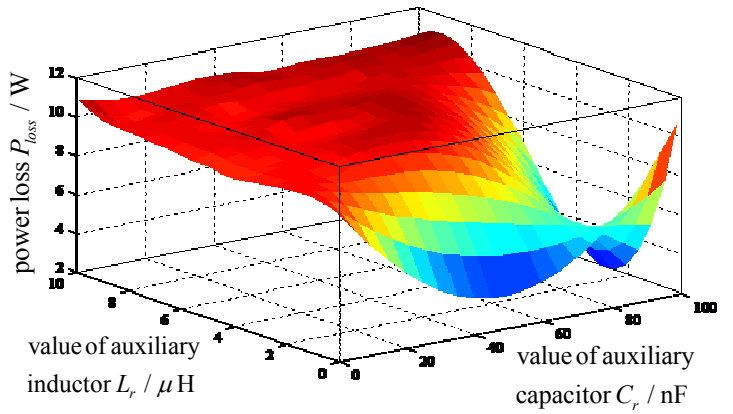

Fig. 6. Obtained response surface.

as an example in this section. This converter is shown in Fig. 4. The additional auxiliary network lies in the dash area, whose passive devices consist an auxiliary inductor $L_{r}$ and an auxiliary capacitor $C_{r}$. The working condition is designed as: input voltage $V_{\text {in }}=40 \mathrm{~V}$, output voltage $V_{o}=100 \mathrm{~V}$, output power $P_{o}=400 \mathrm{~W}$, switching frequency $f_{s}=100 \mathrm{kHz}$, main inductor $L_{m}=45 \mu \mathrm{H}$, output filter capacitor $C_{o}=14 \mu \mathrm{F}$, output capacitor of the main switch $C_{s}=200 \mathrm{pF}$.

The mathematical description form of this example is:

$$
\begin{gathered}
\min P_{\text {loss }}\left(L_{r}, C_{r}\right) \\
\text { s.t. }\left\{\begin{array}{c}
0<L_{r} \leq \frac{V_{o}}{10 f_{s} I_{\text {in }}} \\
0<C_{r} \leq \frac{I_{\text {in }}}{10 f_{s} V_{o}}-C_{s} \\
L_{r} I_{\text {in }}{ }^{2}-\left(C_{r}+C_{s}\right) V_{o}{ }^{2} \geq 0
\end{array}\right.
\end{gathered}
$$

where the first constraint is derived from the principle that the rising time of the auxiliary inductor current is less than $10 \%$ of the switching period [10], while the latter two are derived from the principle that energy stored in the auxiliary inductor is more than that stored in the auxiliary capacitor and output capacitor of the main switch.

The distribution of the experimental sample points is shown in Fig. 5. The range of the axis is calculated according to Eq. (6), and with the constraint of Eq. (6). The sample points cannot fill in the whole space. The response surface is shown in Fig. 6, which represents power loss around the whole space, while only the part that lies in the constraint space possess the ability to guarantee soft-switching (according to Eq. (6) and Fig. 5). Therefore, it is meaningful to research just the constraint response surface, not the whole surface.
TABLE I

Evaluation CRiteria OF THE MOdel

\begin{tabular}{|c|c|c|c|}
\hline Evaluation criteria & $\mathrm{R}^{2}$ & adjusted $\mathrm{R}^{2}$ & $\mathrm{RMSE}$ \\
\hline Value & 1 & 1 & 0.085 \\
\hline
\end{tabular}

TABLE II

Results Of Optimal Variables

\begin{tabular}{|c|c|c|c|}
\hline \multicolumn{2}{|c|}{ Optimization variables } & Space solution & Optimal results \\
\hline \multirow{3}{*}{$1^{\text {st }}$ optimization } & $L_{r} / \mu \mathrm{H}$ & {$[0.1,10]$} & 2.5 \\
& $C_{r} / \mathrm{nF}$ & {$[0.1,99.8]$} & 16.5 \\
& $L_{r} / \mu \mathrm{H}$ & {$[1.2,3.8]$} & 2.4 \\
\multirow{2}{*}{$2^{\text {nd }}$ optimization } & $C_{r} / \mathrm{nF}$ & {$[8.2,24.8]$} & 10 \\
\hline
\end{tabular}

The evaluation criteria of the model are shown in Table I. $\mathrm{R}^{2}$ and adjusted $\mathrm{R}^{2}$ equal 1 , and the RMSE is close to 0 , which demonstrates a good imitative effect.

The parameter optimization with a GA is programed in MATLAB, and the results of the optimal variables are shown in Table II.

From Table II, it can be seen that the optimal solution of the rough optimization is $L_{r}=2.5 \mu \mathrm{H}$ and $C_{r}=16.5 \mathrm{nF}$. Using this result as a center, a smaller solution space which is needed in the second optimization can be obtained (searching the radius is set to $\pm 50 \%$ from the center in this paper). Then accurate optimization is conducted to obtain the final optimal solution: $L_{r}=2.4 \mu \mathrm{H}, C_{r}=10 \mathrm{nF}$. The minimum power loss of the soft-switching converter is $9.4272 \mathrm{~W}$.

Another optimization, with the method proposed in [13], is conducted to play the role of a comparison method, whose optimal parameters are $L_{r}=3.5 \mu \mathrm{H}$ and $C_{r}=22 \mathrm{uF}$. Both of the two groups of parameters are entered into a SPICE model and the simulation results are shown in Fig. 7., Table III, and Table IV (where method 1 is the method proposed in this paper, and method 2 is the method proposed in [13]).

Fig. 7 shows that under both conditions, each output capacitor finishes discharging by means of the resonance with the auxiliary inductor. Intuitively, there is no overlapping area during the turning-on interval (i.e. (a-1), (b-1)), and a tiny overlapping area during the turning-off interval because of the auxiliary capacitor's role as a parallel shunt (i.e. (a-2), (b-2)).

In addition, some of the quantitative parameters shown in Table III are selected to verify the soft-switching effects. $T_{r \text {-on }}$ represents the resonant turn on time while $T_{r \text {-off }}$ represents the resonant turn off time. Since they are influenced by $L_{r}$ and $C_{r}$, they are able to reflect the resonant period. Detailed definitions can be acquired in [11][12]. $I_{\text {peak }}$ represents the peak current during a cycle. Table III shows that both methods possess little $T_{r-o n}$ and $T_{r-o f f}$. This means that the soft-switching takes a little time of the duty cycle, and does not influence the normal operation of the converter. As for the 


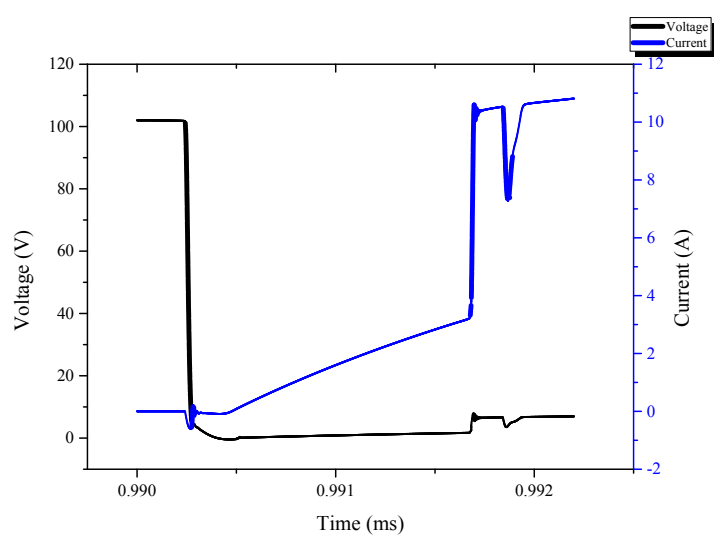

Method 1, turning-on of main switch

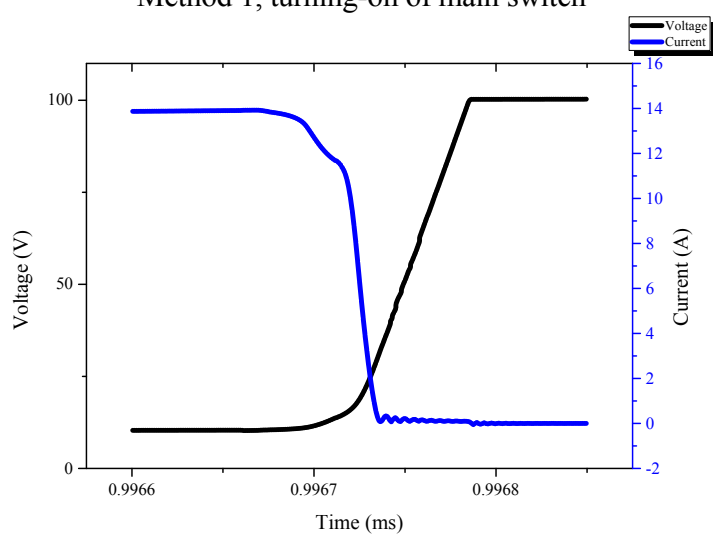

Method 1, turning-off of main switch

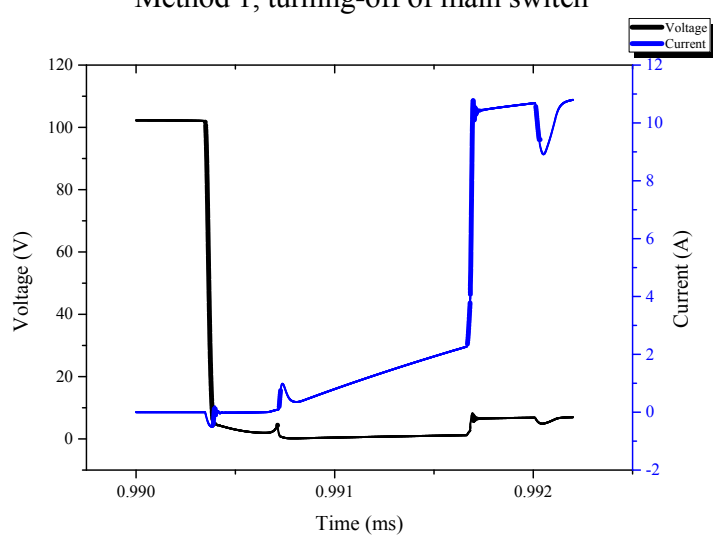

Method 2, turning-on of main switch

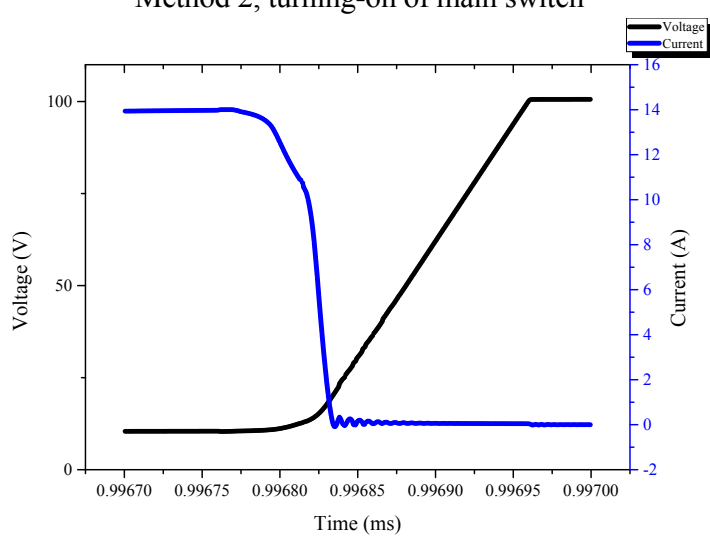

Method 2, turning-off of main switch

Fig. 7. Simulation waveforms of both methods.
TABLE III

QuANTITATIVE COMPARISON OF PARAMETERS

\begin{tabular}{|c|c|c|c|c|}
\hline \multirow{2}{*}{$\begin{array}{c}\text { Circuit } \\
\text { parameters }\end{array}$} & \multicolumn{2}{|c|}{ Simulation } & \multicolumn{2}{c|}{ Experiment } \\
\cline { 2 - 5 } & $\begin{array}{c}\text { Method } \\
1\end{array}$ & $\begin{array}{c}\text { Method } \\
2\end{array}$ & $\begin{array}{c}\text { Method } \\
1\end{array}$ & $\begin{array}{c}\text { Method } \\
2\end{array}$ \\
\hline$T_{r \text {-on }} / \mu \mathrm{s}$ & $1.45 \mathrm{us}$ & $1.35 \mathrm{us}$ & $1.2 \mathrm{us}$ & $1.1 \mathrm{us}$ \\
$T_{r-o f f} / \mu \mathrm{s}$ & $0.103 \mathrm{us}$ & $0.197 \mathrm{us}$ & $0.3 \mathrm{us}$ & $0.25 \mathrm{us}$ \\
$I_{p e a k} / \mathrm{A}$ & $10.81 \mathrm{~A}$ & $10.80 \mathrm{~A}$ & $3.2 \mathrm{~A}$ & $3 \mathrm{~A}$ \\
\hline
\end{tabular}

TABLE IV

Simulation Results Of Both Methods

\begin{tabular}{|c|c|c|}
\hline Circuit parameters & Method 1 & Method 2 \\
\hline$L_{r} / \mu \mathrm{H}$ & 2.4 & 3.5 \\
$C_{r} / \mathrm{nF}$ & 10 & 22 \\
$P_{\text {loss }} / \mathrm{W}$ & 9.4272 & 10.3603 \\
\hline
\end{tabular}

TABLE V

DESIGN SPECIFICATIONS Of EXPERIMENTS

\begin{tabular}{|c|c|}
\hline Parameters & Values \\
\hline Input Voltage & $20 \mathrm{~V}$ \\
Output Voltage & $45 \mathrm{~V}$ \\
Power & $30 \mathrm{~W}$ \\
Switching Frequency & $100 \mathrm{kHz}$ \\
$L_{m}$ & $45 \mu \mathrm{H}$ \\
$C_{o}$ & $14 \mu \mathrm{F}$ \\
& $1.5 \mu \mathrm{H}(\operatorname{method} 1)$ \\
$L_{r}$ & $1.7 \mu \mathrm{H}(\operatorname{method} 2)$ \\
& $7.4 \mathrm{nF}(\operatorname{method} 1)$ \\
$C_{r}$ & $14 \mathrm{nF}(\operatorname{method} 2)$ \\
\hline
\end{tabular}

small $I_{\text {peak }}$, it stands for no extra stress added on the switch.

Table IV shows that method 1 is better. Its power loss is $9.01 \%$ less than that of method 2 . In addition, comparing the method of exhaustion used in [13], the method proposed in this paper, which uses a GA, possesses better searching ability and stronger robustness.

Furthermore, a $30 \mathrm{~W}$ experimental prototype circuit is built and tested to verify the advantages of the proposed method. Specifications of the experiments are shown in Table V. The experimental results are shown in Fig. 8 and Table III.

Fig. 8 shows that both $v_{S}$ (i.e. the voltage between the drain and source of the main switch) have fallen down to zero before turning on. This results in no overlapping area between $v_{S}$ and $i_{S}$ (i.e. the current of the main switch). Meanwhile, $C_{r}$ undertakes part of the current when turning off, which decreases the power loss of turning off. Similar to the simulation results, the experimental results show that both methods can realize soft-switching to some extent. 


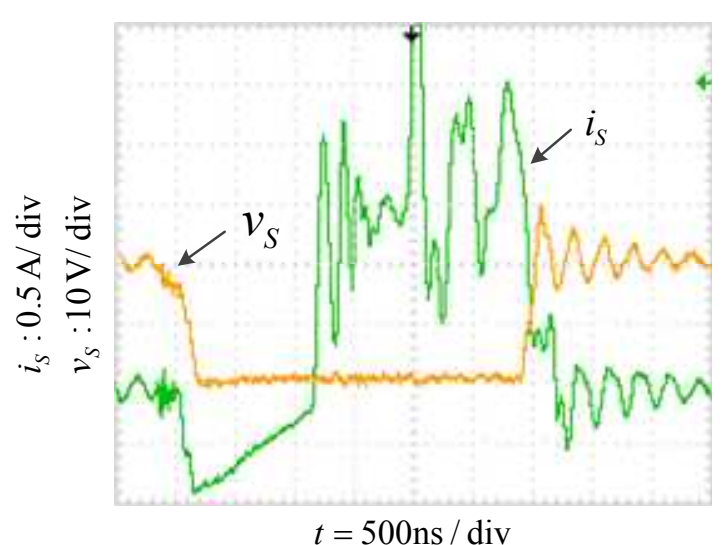

(a)

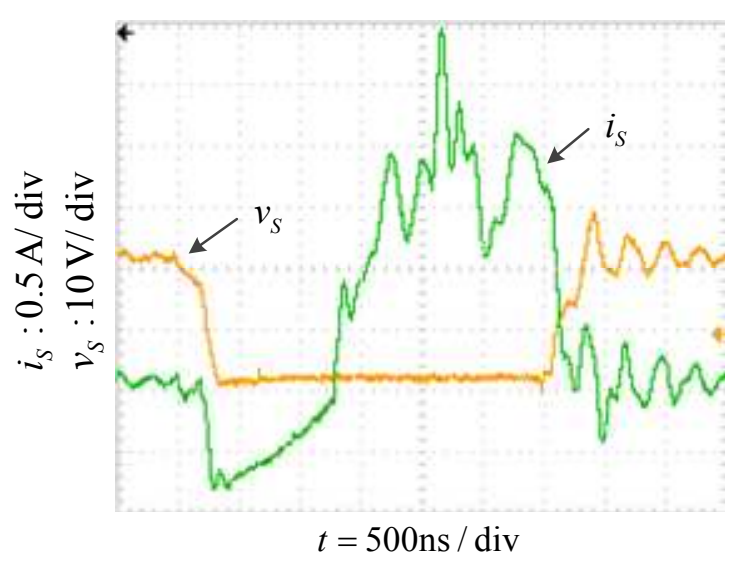

(b)

Fig. 8. Experiment waveforms of both methods. (a) Method 1, switching period of main switch. (b) Method 2, switching period of main switch.

Table III also verifies the soft-switching of both methods from the quantitative perspective.

In addition to measuring the voltage and current of the input and output, the efficiency can also be calculated. The efficiency of method 1 is $85.84 \%$, while that of method 2 is $81.66 \%$. This proves that the proposed method is better.

\section{CONCLUSION}

This paper uses a soft-switching converter as a background, introduces the method and steps of the proposed soft-switching parameters optimization with a RSM model, a SPICE model, and a GA. Example results demonstrates that the proposed RSM model possesses a higher fitting accuracy. This can narrow the wide solution space reasonably and effectively, and increase the algorithm efficiency and global optimization effect. The GA possesses a better optimization effect and global search when compared to the method of utilizing engineering experience or exhaustion. In applications where the here are more soft-switching parameters, the method of utilizing engineering experience suffers from greater risks and consumes more resources. This manifests the advantages of the
GA. Furthermore, by considering soft-switching in a wide operation range (e.g. a wide range of input line, output current, step-up ratio, duty ratio, etc.), or restrictions in the converter volume and cost, conventional parameters design methods face greater challenges, while the GA method can be expanded to a multiple objective optimization method. In conclusion, the RSM-SPICE-GA method has engineering value and significance in the area of soft-switching converter parameter design.

\section{REFERENCES}

[1] G. Cao, and H. J. Kim, "Improved bridgeless interleaved Boost PFC rectifier with optimized magnetic utilization and reduced sensing noise," Journal of Power Electronics, Vol. 14, No. 5, pp. 815-826, Sep. 2014.

[2] D. K. Jeong, M. H. Ryu, H. G. Kim, and H. J. Kim, "Optimized design of bi-directional dual active bridge converter for low-voltage battery charger," Journal of Power Electronics, Vol. 14, No. 3, pp. 468-477, May 2014.

[3] M. S. Song, Y. D. Son, and K. H. Lee, "Non-isolated bidirectional soft-switching SEPIC/ZETA converter with reduced ripple currents," Journal of Power Electronics, Vol. 14, No. 4, pp. 649-660, Jul. 2014.

[4] P. Radika, "A high efficiency DC-DC Boost converter with passive regenerative snubber," Journal of Electrical Engineering \& Technology, Vol. 9, No. 2, pp. 501-507, Mar. 2014.

[5] N. Altintas, "A novel single phase soft switched PFC converter," Journal of Electrical Engineering \& Technology, Vol. 9, No. 5, pp. 1592-1601, Sep. 2014.

[6] G. Cao and H. Kim, "A novel circuit for characteristics measurement of $\mathrm{SiC}$ transistors," Journal of Electrical Engineering \& Technology, Vol. 9, No. 4, pp. 1332-1342, Jul. 2014.

[7] N. Altintas, A. F. Bakan, and I. Aksoy, "A novel ZVT-ZCT-PWM Boost converter," IEEE Trans. Power Electron., Vol. 29, No. 1, pp. 256-265, Jan. 2014.

[8] I. Aksoy, H. Bodur, and A. F. Bakan, "A new ZVT-ZCT-PWM DC-DC converter," IEEE Trans. Power Electron., Vol. 25, No. 8, pp. 2093-2105, Aug. 2010.

[9] M. Rezvanyvardom, E. Adib, H. Farzanehfard, "New interleaved zero-current switching pulse-width modulation boost converter with one auxiliary switch," IET Power Electron., Vol. 4, No. 9, pp. 979-983, Mar. 2011.

[10] D. Y. Jung, Y. H. Ji, S. H. Park, Y. C. Jung, and C. Y. Won, "Interleaved soft-switching Boost converter for photovoltaic power-generation system," IEEE Trans. Power Electron., Vol. 26, No. 4, pp. 1137-1145, Apr. 2011.

[11] K. M. Smith, Jr., K. M. Smedley, "Engineering design of lossless passive soft switching methods for PWM converters. I. with minimum voltage stress circuit cells," IEEE Trans. Power Electron., Vol. 16, No. 3, pp. 336-344, May 2001.

[12] K. M. Smith, Jr., K. M. Smedley, "Engineering design of lossless passive soft switching methods for PWM converters. II. Non-minimum voltage stress circuit cells," IEEE Trans. Power Electron., Vol. 17, No. 6, pp. 864-873, Nov. 2012. 
[13] I. Matsuura, K. M. Smith, K. M. Smedley, "A comparison of active and passive soft switching methods for PWM converters," Proceedings of the 29th Annual IEEE Power Electronics Specialists Conference, pp. 94-100, 1998.

[14] S. M. Ayob, Z. Salam, and N. A. Azli, "A new optimum design for a single input fuzzy controller applied to DC to AC converters," Journal of Power Electronics, Vol. 10, No. 3, pp.306-312, May 2010.

[15] G. Mahendran, M. Sathiskumar, S. Thiruvenkadam, and L. Lakshminarasimman, "Multi-objective Unbalanced Distribution Network Reconfiguration through Hybrid Heuristic Algorithm," Journal of Electrical Engineering \& Technology, Vol. 8, No. 2, pp. 215-222, Mar. 2013.

[16] F. Dufosse, K. Kaya, and B. Ucar, "Bipartite matching heuristics with quality guarantees on shared memory parallel computers," IEEE $28^{\text {th }}$ Parallel and Distributed Processing Symposium, pp. 540-549, 2014.

[17] Y. Cui, M. Chinthavali, and L. M. Tolbert, "Temperature dependent Pspice model of silicon carbide power MOSFET," Applied Power Electronics Conference and Exposition, pp. 1698-1704, 2012.

[18] R. Fu, A. Grekov, J. Hudgins, A. Mantooth, and E. Santi, "Power SiC DMOSFET model accounting for nonuniform current distribution in JFET region," IEEE Trans. Industry Applications, Vol. 48, No. 1, pp. 181-190, Jan. 2012.

[19] V. Christophe, D. Olivier, and L. Jacques, "A response surface methodology approach to study the influence of specifications or model parameters on the multiobjective optimal design of isolated DC-DC converters," IEEE Trans. Power Electron., Vol. 27, No. 7, pp. 3383-3395, Jul. 2012.

[20] D. Cai, D. Shi, and J. Chen, "Probabilistic load flow computation using Copula and Latin hypercube sampling", IET Generation, Transmission \& Distribution, Vol. 8, No. 9, pp. 1539-1549, Sep. 2014.

[21] K. Yang, G. Ouyang, and H. Chen, "Optimization of unit commitment of marine power system using improved genetic algorithm," Control Theory \& Applications, Vol. 28, No. 5, pp. 722-726, May 2011.

[22] R. Streit, and D. Tollik, "High efficiency telecom rectifier using a novel soft-switched boost-based input current shaper," Proceedings of the $13^{\text {th }}$ International Telecommunications Energy Conference, pp. 720-726, 1991.

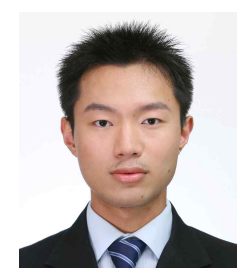

Shuai Liu received his B.S. degree in Electrical Engineering from Tongji University, Shanghai, China, in 2010, where he is presently working toward his Ph.D. degree in Control Theory and Engineering. Since autumn of 2010, he has been with the Laboratory of New Energy and Electrical Equipment, Tongji University. His current research interests include the development of soft-switching converters for electric vehicles, including topology innovations, control strategies, and parameter optimization.

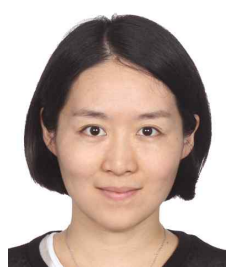

Li Wei received her B.S. degree in Electrical Engineering and her Ph.D. degree in Control Theory and Engineering from Tongji University, Shanghai, China, in 2004 and 2010, respectively. She worked in the Postdoctoral Center of Computer Science and Engineering from 2010 to 2013, and she is presently an Assistant Professor in the Department of Electrical Engineering, Tongji University. Since 2005, she has been with the Laboratory of New Energy and Electrical Equipment, Tongji University. Her current research interests include the modeling and control of converters, large-scale super-capacitor energy storage systems and their application to metro systems. In these research fields, she has been leading several industrial and government projects.

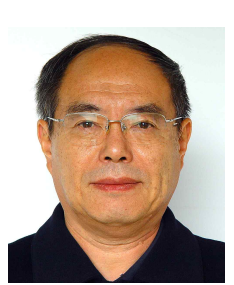

Yicheng Zhang received his B.S. degree in Electric Drives for Locomotives and his M.S. degree in Railway Electrical and Automotive Traction from the former Shanghai Railway University, Shanghai, China, in 1982 and 1987, respectively. He received his Ph.D. degree in Electrical Apparatus from Fuzhou University, Fuzhou, China, in 1993. From 1982 to 1999, he worked in the Department of Electrical Engineering in the former Shanghai Railway University. Since 2000, he has been working as a Professor in the Department of Electrical Engineering, Tongji University, Shanghai China. He founded the Lab of New Energy and Electrical Equipment, Tongji University, in 2002. His current research interests include the development of $\mathrm{dc} / \mathrm{dc}$ converters for fuel cell cars, electromagnetic components design, EMC solutions for $\mathrm{dc} / \mathrm{dc}$ converters, and super-capacitor energy storage systems and their management. In these research fields, he has been leading several industrial and government projects.

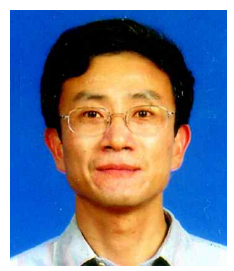

Yongtao Yao received his B.S. degree in Electric Drives for Locomotives from the former Shanghai Railway University, Shanghai, China, in 1984. From 1984 to 1999, he worked in the Department of Electrical Engineering, former Shanghai Railway University. Since 2000, he has been working as a Senior Engineer in the Department of Electrical Engineering, Tongji University, Shanghai China. He cofounded the Lab of New Energy and Electrical Equipment, Tongji University, in 2002. His current research interests include the development of $\mathrm{dc} / \mathrm{dc}$ converters for fuel cell cars, modeling and control, multiphase parallel techniques for converters, electromagnetic components design, and EMC solutions for dc/dc converters. In these research fields, he has been leading several industrial and government projects. 